\title{
Keratin 19 mRNA is detectable by RT-PCR in Iymph nodes of patients without breast cancer
}

\section{Sir}

We were interested to read the paper on the use of cytokeratin 19 (K19) mRNA by reverse transcription polymerse chain reaction (RT- PCR) combined with Southern blotting for the detection of lymph node micrometastasis in breast cancer patients by Schoenfeld et al (1996). The authors have reported that, among 75 histologically node-negative breast cancer patients, $23(30.6 \%)$ demonstrate K19 mRNA in their lymph nodes whereas none of 28 control lymph nodes without epithelial malignancy show K19 expression.

This report and another by Traweek et al (1993) are in sharp contrast with our data in that K19 mRNA is readily detected by RT-PCR in 31 of 40 (77.5\%) lymph nodes from five of eight patients with benign bowel diseases, none of whom had any signs of an epithelial malignancy. We also found that two of these same eight had K19-positive bone marrow aspirates. Our methods involve extremely careful dissection of lymph nodes before cutting any epithelial tissue to avoid epithelial cell contamination from surgical gloves or dissection equipment. RNA extractions were performed, including blank samples, so that reagent contamination could not account for positive results. Our PCR strategy allows discrimination of K19 cDNA-derived PCR products from K19 genomic DNA- or K19 pseudogene-derived PCR products, methodologies of which have recently been published by Gunn et al (1996). Furthermore, we showed that the 31 lymph nodes that expressed K19 mRNA did not express keratin 20, a gene expressed highly by the epithelial cells of the gastrointestinal tract only) ruling out epithelial cell contamination as the source of K19 mRNA in these lymph nodes. Among 35 breast cancer patients so far studied, 17 were histologically node negative whereas 18 were histologically node positive. Ninety-five of 143 lymph nodes (66.4\%) from the former group and 128 of 166 lymph nodes (77.1\%) from the latter group were found to be K19 positive by
RT-PCR. From these findings we have concluded that a low level of K19 mRNA is expressed in most lymph nodes, and that these data concur with previous reports by Krisman et al (1995), Adams et al (1995) and Burchill et al (1995).

\section{K Yun \\ Department of Pathology, University of Otago Medical School, Dunedin, New Zealand}

J Gunn, AEH Merrie and LV Phillips

Department of Surgery, University of Otago Medical School, Dunedin, New Zealand

$J L$ McCall

Department of Surgery, Auckland University Medical School, Auckland, New Zealand

\section{REFERENCES}

Adams MD, Kerlavage AR, Fleischmann RD, Fuldner RA, Bult CJ, Lee NH, Kirkness EF, Weinstock KG, Gocayne JD, White O et al (1995) Initial assessment of human gene diversity and expression patterns based upon 83 million nucleotides of cDNA sequence. Nature 377: 3-174

Burchill SA, Bradbury MF, Pittman K, Southgate J, Smith B and P Selby (1995) Detection of epithelial cancer cells in peripheral blood by reverse transcriptase-polymerase chain reaction. Br J Cancer 71: 278-281

Gunn J, McCall JL, Yun K and Wright PA (1996) Detection of micrometastases in colorectal cancer patients by K19 and K20 reverse-transcription polymerase chain reaction. Lab Invest 75: 611-616

Krismann M, Todt B, Schroder J, Gareis D, Muller KM, Seeber S and Schutte J (1995) Low specificity of cytokeratin 19 reverse transcriptase-polymerase chain reaction analyses for detection of hematogenous lung cancer dissemination. J Clin Oncol 13: 2769-2775

Schoenfeld A, Luqmani Y, Sinnett HD, Shousha S and Coombes RC (1996) Keratin 19 mRNA measurement to detect micrometastases in lymph nodes in breast cancer patients. Br J Cancer 74: 1639-1642

Traweek ST, Liu J and H Battifora (1993) Keratin gene expression in non-epithelial tissues. Am J Pathol 142: 1111-1118

\section{Keratin 19 mRNA is detectable by RT-PCR in Iymph nodes of patients without breast cancer - reply}

\section{Sir}

We agree that K19 mRNA is detectable in normal lymph nodes (from patients without any epithelial malignancy) by reverse transcription polymerase chain reaction (RT-PCR) after many cycles of amplification. In our paper (Schoenfeld et al, 1994), we described the methodology and demonstrated a 'cut-off' of 40 cycles of amplification beneath which all normal lymph nodes remained negative for K19 mRNA; those above this level may be taken to indicate lymph node metastases. In our studies, this "cutoff' occurred after 40 cycles of RT-PCR and Southern hybridization. 
A second amplification, using nested primers, resulted in the appearance of mRNA-derived K19 product in normal nodes as well as nodes from cancer patients. Thus, there is a low level of K19 mRNA in normal nodes, and the potential of RT-PCR in the staging of breast cancer is therefore limited by the specificity of the tumour marker.
RC Coombes

Cancer Research Campaign Laboratories,

Department of Medical Oncology, Charing Cross Medical

School, London W6 8RP, UK

\section{REFERENCES}

Schoenfeld A, Luqmani Y, Smith D, O'Reilly S, Shousha S, Sinnett HD and Coombes RC (1994) Detection of breast cancer micrometastases in axillary lymph nodes by using polymerase chain reaction. Cancer Res 54: 2986-2990

\section{Mutations at codon 974 of the DPYD gene are a rare event}

Sir

We regret to inform you of an error made in a recently published article in the British Journal of Cancer (75: 178-179). 'Mutations at codon 974 of the DPYD gene are a rare event' from Ridge et al (1997). In this study, Ridge et al attempted to determine the frequency of a mutation originally described by our laboratory in 1995 (abstract presented at the American Association of Cancer Research, AACR). Unfortunately, the authors of this study misinterpreted the position of the mutation [A (control) to T (mutation)] originally localized at position 2921 in the open reading frame of dihydropyrimidine dehydrogenase cDNA. When translated, this mutation results in an Asp to Val substitution corresponding to codon 949 , not to codon 974 as reported by Ridge et al. The position of the mutation reported in the AACR abstract was based on a complete human DPD cDNA amplified using primers designed against bovine liver cDNA (Figure 1). Both the original numbering system and the codon number (949) were presented at the 1995 AACR meeting (Albin N et al (1995) Proc Am Assoc Cancer Res 36: 211).

After a careful review of this article, we have determined that Ridge et al assigned the first adenosine nucleotide in the initiating methionine as the number 1 position. This resulted in the incorrect assignment of codon 974 as the site of the mutation (Figure 1). We concur with Ridge et al that the frequency of this mutation should be determined in the population and will therefore forward a copy of this letter to Ridge et al, along with an offer to provide any information necessary to correct this study.

\author{
RB Diasio \\ Department of Pharmacology and Toxicology, \\ Comprehensive Cancer Centre, \\ University of Alabama at Birmingham \\ Birmingham, AL 35294, USA

\section{MR Johnson} \\ Department of Pharmacology and Toxicology, \\ University of Alabama at Birmingham, \\ Birmingham, AL 35294, USA

\section{N Albin} \\ Department of Pharmacology and Toxicology, \\ University of Alabama at Birmingham, \\ Birmingham, AL 35294, USA
}

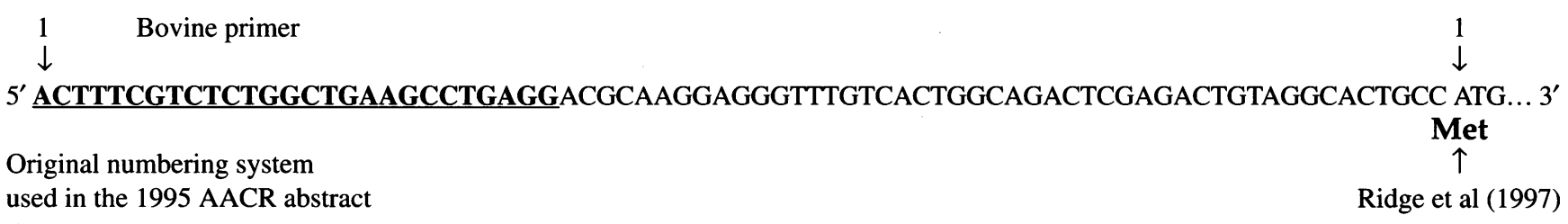

Figure 1 The bovine primer (bold and underlined) used to PCR amplify the $5^{\prime}$ end of human lymphocyte DPD cDNA. The original numbering system assigned the $5^{\prime}$ end of the bovine primer as the number 1 position, with the mutation ultimately corresponding to position 2921 , as originally presented in the abstract. Ridge et al (1997) assigned the first adenosine nucleotide in the initiating methionine as the number 1 position and therefore localized the mutation to the wrong codon (974 instead of 949) 